\title{
Publisher's Note: Empirical analysis of the lane formation process in bidirectional pedestrian flow [Phys. Rev. E 94, 032304 (2016)]
}

Claudio Feliciani and Katsuhiro Nishinari

(Received 8 June 2018; published 12 June 2018)

DOI: 10.1103/PhysRevE.97.069901

This paper was published online on 7 September 2016 with an error in Fig. 16 and an error in the text on page 13. On page 13, the first full sentence after Eq. (7) should read as "The order parameter is always positive, even in the extreme case of random movement and becomes 0 only for vertically aligned patterns." The figure and text have been corrected as of 8 June 2018. The text and figure are incorrect in the printed version of the journal; therefore, for the benefit of the print readership, the figure and its caption are replicated below.

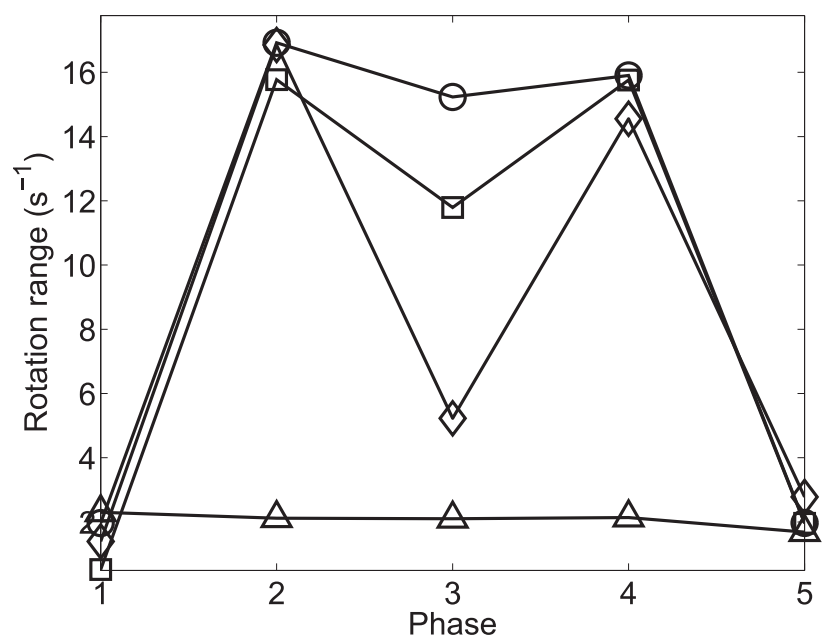

FIG. 16. Rotation range (from minimum to maximum) during the different phases and for each configuration. The following legend is used: circle for the $5 / 1$ configuration, square for the $4 / 2$ configuration, diamond for the $3 / 3$ configuration, and triangle for the $6 / 0$ configuration. 